\title{
Statistical structures on tangent bundles and tangent Lie groups
}

\author{
Esmaeil Peyghan ${ }^{1}$ (D), Davood Seifipour ${ }^{1}$ (D), Aydin Gezer*2 (D) \\ ${ }^{1}$ Department of Mathematics, Faculty of Science, Arak University, Arak, 38156-8-8349, Iran \\ ${ }^{2}$ Ataturk University, Faculty of Science, Department of Mathematics, 25240, Erzurum-Turkey
}

\begin{abstract}
Let $T M$ be a tangent bundle over a Riemannian manifold $M$ with a Riemannian metric $g$ and $T G$ be a tangent Lie group over a Lie group with a left-invariant metric $g$. The purpose of the paper is two folds. Firstly, we study statistical structures on the tangent bundle $T M$ equipped with two Riemannian g-natural metrics and lift connections. Secondly, we define a left-invariant complete lift connection on the tangent Lie group $T G$ equipped with metric $\widetilde{g}$ introduced in [F. Asgari and H. R. Salimi Moghaddam, On the Riemannian geometry of tangent Lie groups, Rend. Circ. Mat. Palermo II. Series, 2018] and study statistical structures in this setting.
\end{abstract}

Mathematics Subject Classification (2020). 53C07, 53C25, 62B10

Keywords. metric, statistical structure, tangent bundle, tangent Lie group, lift connection

\section{Introduction}

Information geometry is a combination and interaction of differential geometry and statistics. Also, it is an important and useful bridge between applicable and pure sciences. In this area, we use and extend the methods of differential geometry in probability theory. The mathematical point of view of information geometry started by Rao in [14]. He showed that a statistical model should be as a differential Riemannian manifold via the Fisher information matrix. This means that we can define a Riemannian metric in the space of probability distributions. In fact, the information geometry is study of natural geometric structures by the families of probability distributions. The main objects in this area are statistical connections and statistical manifolds and their applications such as in computer science and physics. The geometry of statistical manifolds has been applied to various fields of information science, information theory, neural networks, statistical mechanics (see $[2,5,16]$, for instance). In fact, a statistical manifold is a manifold whose points are probability distributions and so statistical manifolds provide geometric model of probability distributions.

\footnotetext{
*Corresponding Author.

Email addresses: e-peyghan@araku.ac.ir (E. Peyghan),d-seifipour@phd.araku.ac.ir (D. Seifipour), agezer@atauni.edu.tr (A. Gezer)

Received: 11.11.2019; Accepted: 17.03.2021
} 
A pair $(g, \nabla)$ on a differentiable manifold $M$ is a statistical structure if $\nabla g$ is totally symmetric, where $g$ is a (pseudo-) Riemannian metric and $\nabla$ is a torsion free linear connection. A manifold with a statistical structure is called a statistical manifold. A (pseudo-) Riemannian manifold $(M, g)$ together with Levi-Civita connection $\nabla$ of $g$ is a typical example of statistical manifold. In other words, statistical manifolds can be regarded as generalizations of (pseudo-) Riemannian manifolds.

In this paper, we mostly concern with statistical structures on a tangent bundle $T M$ and a tangent Lie group $T G$ with respect to the lift connections. Recently, some mathematicians studied the statistical structures on the tangent bundle of a smooth manifolds (see $[4,9,11]$, for instance).

The organization of this paper is as follows. In Section 3, we consider two Riemannian $g$-natural metrics on $T M$. One of them is $G_{a, b, c}=a g^{s}+b g^{c}+c g^{v}$, generated by the classical lifts the Sasaki metric $g^{s}$, the complete lift metric $g^{c}$ and the vertical lift $g^{v}$ of Riemannian metric $g$ and the another is the Cheeger-Gromoll metric $g_{C G}$. Then, we study that under what conditions $\left(G_{a, b, c}, \nabla^{h}\right)$ is a Codazzi pair for $T M$. Also, we prove that $\left(G_{a, b, c}, \nabla^{h}\right)$ is a Codazzi pair on $T M$ if and only if $\nabla$ is a metric connection with respect to $g$, especially in a particular case $\left(G_{a, 0,0}, \nabla^{h}\right)$ is a Codazzi pair on $T M$ if and only if the pair $(g, \nabla)$ is a Codazzi pair on $M$. Furthermore, we prove that if $(g, \nabla)$ is a Codazzi pair on $M$, then $\left(g_{C G}, \nabla^{h}\right)$ is not a Codazzi pair on $T M$. Also, accordingly to the concept of complete connection we show that $\left(T M, G_{a, b, c}, \nabla^{c}\right)$ is a statistical manifold if and only if $\nabla$ is a flat Levi-Civita connection, and in this case $\left(T M, G_{a, b, c}, \nabla^{c}\right)$ reduces to the Riemannian manifold. In Section 4, we study the concept of tangent Lie group TG. Also in this section we consider a left-invariant metric $\bar{g}$ on tangent Lie group $T G$, then we study that under what conditions $\left(\bar{g}, \nabla^{c}\right)$ can provide a Codazzi pair on $T G$, whenever $(g, \nabla)$ is a Codazzi pair for $G$, and $g$ is a left-invariant metric and $\nabla$ is a left-invariant connection.

\section{Preliminaries}

Let $M$ be an $n$-dimensional differentiable manifold with a linear connection $\nabla$ and denote by $\pi: T M \rightarrow M$ its tangent bundle with fibres the tangent spaces to $M$. Then, $T M$ is a $2 n$-dimensional smooth manifold. Let $P$ be an arbitrary point in $U$ in $M$ whose coordinates are $\left(x^{i}\right)$. On $T M$, the system of local coordinates $\left(\pi^{-1}(U), x^{i}, x^{\bar{i}}=y^{i}\right)$, $\bar{i}=n+i=n+1, \ldots, 2 n$, where $\left(y^{i}\right)$ is the cartesian coordinates in each tangent space $T_{p} M$ at $p$ with respect to the natural base $\left\{\left.\frac{\partial}{\partial x^{i}}\right|_{p}\right\}$, is induced from a system of local coordinates $\left(U, x^{i}\right)$ in $M$. Also, the set $\left\{\left.\frac{\partial}{\partial x^{i}}\right|_{p},\left.\frac{\partial}{\partial y^{i}}\right|_{p}\right\}$ is the natural basis of $T_{p} T M$. Summation over repeated indices is always implied.

The various lifts (complete, horizontal and vertical lifts, respectively) of a vector field $X=X^{i} \partial_{i}$ on $M$ to $T M$ are defined as follows

$$
X^{c}=X^{i} \frac{\partial}{\partial x^{i}}+y^{a}\left(\partial_{a} X^{i}\right) \frac{\partial}{\partial y^{i}}, \quad X^{h}=X^{i} \frac{\partial}{\partial x^{i}}-y^{a} \Gamma_{a i}^{k} X^{i} \frac{\partial}{\partial y^{k}}, \quad X^{v}=X^{i} \frac{\partial}{\partial y^{i}},
$$

where $\Gamma_{s k}^{i}$ are the coefficients of the linear connection $\nabla$. Recall that the Lie brackets of horizontal and vertical vector fields are given by [17]

$$
\begin{aligned}
& {\left[X^{h}, Y^{h}\right]=[X, Y]^{h}-(R(X, Y) y)^{v},} \\
& {\left[X^{h}, Y^{v}\right]=\left(\nabla_{X} Y\right)^{v}-T(X, Y)^{v},} \\
& {\left[X^{v}, Y^{v}\right]=0 .}
\end{aligned}
$$

It is known that $T T M$ can be decompose to $H T M \oplus V T M$, where $H T M$ is spanned by $\left\{\frac{\delta}{\delta x^{i}}:=\left(\frac{\partial}{\partial x^{i}}\right)^{h}=\frac{\partial}{\partial x^{i}}-y^{k} \Gamma_{k i}^{j} \frac{\partial}{\partial y^{j}}\right\}$ and $V$ is spanned by $\left\{\frac{\partial}{\partial y^{i}}:=\left(\frac{\partial}{\partial x^{i}}\right)^{v}\right\}$. For simplicity we write $\partial_{i}, \delta_{i}$ and $\partial_{\bar{i}}$ instead of $\frac{\partial}{\partial x^{i}}, \frac{\delta}{\delta x^{i}}$ and $\frac{\partial}{\partial y^{i}}$, respectively. 
Let $(M, g)$ be a Riemannian manifold. The Riemanian $g$-natural metric of the form $G_{a, b, c}=a g^{s}+b g^{c}+c g^{v}$, generated by the classical lifts the Sasaki metric $g^{s}$, the complete lift metric $g^{c}$ and the vertical lift $g^{v}$ of $g$ is defined by

$$
\begin{aligned}
& G_{a, b, c}\left(X^{h}, Y^{h}\right)=(a+c) g(X, Y) \\
& G_{a, b, c}\left(X^{v}, Y^{h}\right)=G_{a, b, c}\left(X^{h}, Y^{v}\right)=b g(X, Y) \\
& G_{a, b, c}\left(X^{v}, Y^{v}\right)=a g(X, Y)
\end{aligned}
$$

for all vector fields $X, Y$ on $M$, where $a, b$ and $c$ are constants satisfying $a>0$ and $a(a+c)-b^{2}>0$ [1] (see also [6]). Also, there exists another well-known lift metric: The Cheeger-Gromoll metric $g_{C G}$ is given by [8]

$$
\begin{aligned}
& g_{C G}\left(X^{h}, Y^{h}\right)=g(X, Y), \\
& g_{C G}\left(X^{h}, Y^{v}\right)=0, \\
& g_{C G}\left(X^{v}, Y^{v}\right)=\frac{1}{1+r^{2}}\{g(X, Y)+g(X, y) g(Y, y)\},
\end{aligned}
$$

for all vector fields $X, Y$ on $M$, where $r$ denotes $r=\sqrt{g(y, y)}$.

If we write $d s^{2}=g_{i j} d x^{i} d x^{j}$ for the (pseudo-) Riemannian metric on $M$ given by $g$, then the Riemannian metrics on $T M$ given by $G_{a, b, c}$ and $g_{C G}$ respectively are

$$
\begin{aligned}
G_{a, b, c} & =(a+c) g_{i j} d x^{i} d x^{j}+2 b g_{i j} \delta y^{i} d x^{j}+a g_{i j} \delta y^{i} \delta y^{j}, \\
g_{C G} & =g_{i j} d x^{i} d x^{j}+\frac{1}{1+r^{2}}\left(g_{i j}+y^{s} y^{t} g_{i s} g_{j t}\right) \delta y^{i} \delta y^{j},
\end{aligned}
$$

where $\delta y^{i}=d y^{i}+y^{s} \Gamma_{j s}^{i} d x^{j}$ being Christoffel symbols formed with $g$.

Let $M$ be a differentiable manifold with a linear connection $\nabla$. The horizontal lift connection $\nabla^{h}$ and the complete lift connection $\nabla^{c}$ are respectively defined by [17]

$$
\begin{gathered}
\nabla_{X^{h}}^{h} Y^{h}=\left(\nabla_{X} Y\right)^{h}, \quad\left(\nabla_{X^{h}}^{h} Y^{v}\right)=\left(\nabla_{X} Y\right)^{v}, \quad \nabla_{X^{v}}^{h} Y^{h}=\nabla_{X^{v}}^{h} Y^{v}=0, \\
\nabla_{X^{h}}^{c} Y^{h}=\left(\nabla_{X} Y\right)^{h}+(R(y, X) Y)^{v}, \nabla_{X^{h}}^{c} Y^{v}=\left(\nabla_{X} Y\right)^{v}, \quad \nabla_{X^{v}}^{c} Y^{h}=\nabla_{X^{v}}^{c} Y^{v}=0,
\end{gathered}
$$

for all vector fields $X, Y$ on $M$.

\section{Statistical structures on the tangent bundle with respect to the lift connections}

Let $\nabla$ be an arbitrary linear connection on a (pseudo-) Riemannian manifold $(M, g)$. Given a pair $(\nabla, g)$, we construct the $(0,3)$ - tensor fields $F$ by

$$
F(X, Y, Z):=\left(\nabla_{Z} g\right)(X, Y) .
$$

Clearly, $F(X, Y, Z)=F(Y, X, Z)$, due to symmetry of $g$. The tensor field $F$ is sometimes referred to as the cubic form associated to the pair $(\nabla, g)[7]$.

For a symmetric bilinear form $\rho$ on a manifold $M$, we call $(\nabla, \rho)$ a Codazzi pair, if the covariant derivative $(\nabla \rho)$ is (totally) symmetric in $X, Y, Z[15]$ :

$$
\left(\nabla_{Z} \rho\right)(X, Y)=\left(\nabla_{X} \rho\right)(Z, Y)=\left(\nabla_{Y} \rho\right)(Z, X) .
$$

In terms of the cubic form $F$, this condition is stated as

$$
F(X, Y, Z)=F(Z, Y, X)=F(Z, X, Y),
$$

i.e., the condition $(\nabla, g)$ being Codazzi pair is equivalent to $F$ being totally symmetric in all of its indices. Now, we search the conditions under which the pair $\left(\nabla^{h}, G\right)$ is a Codazzi 
pair on $T M$. For using later on, we give

$$
\begin{aligned}
& \left(\nabla_{\delta_{i}}^{h} G_{a, b, c}\right)\left(\delta_{j}, \delta_{k}\right)=\delta_{i} G_{a, b, c}\left(\delta_{j}, \delta_{k}\right)-G_{a, b, c}\left(\nabla_{\delta_{i}}^{h} \delta_{j}, \delta_{k}\right)-G_{a, b, c}\left(\delta_{j}, \nabla_{\delta_{i}}^{h} \delta_{k}\right) \\
& =\delta_{i}(a+c) g_{j k}-G_{a, b, c}\left(\left(\nabla_{\partial_{i}} \partial_{j}\right)^{h},\left(\partial_{k}\right)^{v}\right)-G_{a, b, c}\left(\left(\partial_{j}\right)^{h},\left(\left(\nabla_{\partial_{i}} \partial_{k}\right)^{h}\right)\right. \\
& =(a+c) \partial_{i} g_{j k}-(a+c) g\left(\nabla_{\partial_{i}} \partial_{j}, \partial_{k}\right)-(a+c) g\left(\partial_{j}, \nabla_{\partial_{i}} \partial_{k}\right) \\
& =(a+c) \nabla_{i} g_{j k}, \\
& \left(\nabla_{\delta_{i}}^{h} G_{a, b, c}\right)\left(\delta_{j}, \partial_{\bar{k}}\right)=\delta_{i} G_{a, b, c}\left(\delta_{j}, \partial_{\bar{k}}\right)-G_{a, b, c}\left(\nabla_{\delta_{i}}^{h} \delta_{j}, \partial_{\bar{k}}\right)-G_{a, b, c}\left(\delta_{j}, \nabla_{\delta_{i}}^{h} \partial_{\bar{k}}\right) \\
& =\delta_{i} b g_{j k}-G_{a, b, c}\left(\left(\nabla_{\partial_{i}} \partial_{j}\right)^{h},\left(\partial_{k}\right)^{v}\right)-G_{a, b, c}\left(\left(\partial_{j}\right)^{h},\left(\left(\nabla_{\partial_{i}} \partial_{k}\right)^{v}\right)\right. \\
& =b \partial_{i} g_{j k}-b g\left(\nabla_{\partial_{i}} \partial_{j}, \partial_{k}\right)-b g\left(\partial_{j}, \nabla_{\partial_{i}} \partial_{k}\right) \\
& =b \nabla_{i} g_{j k}, \\
& \left(\nabla_{\delta_{i}}^{h} G_{a, b, c}\right)\left(\partial_{\bar{j}}, \partial_{\bar{k}}\right)=\delta_{i} G_{a, b, c}\left(\partial_{\bar{j}}, \partial_{\bar{k}}\right)-G_{a, b, c}\left(\nabla_{\delta_{i}}^{h} \partial_{\bar{j}}, \partial_{\bar{k}}\right)-G_{a, b, c}\left(\partial_{\bar{j}}, \nabla_{\delta_{i}}^{h} \partial_{\bar{k}}\right) \\
& =\delta_{i} c g_{j k}-G_{a, b, c}\left(\left(\nabla_{\partial_{i}} \partial_{j}\right)^{v},\left(\partial_{k}\right)^{v}\right)-G_{a, b, c}\left(\left(\partial_{j}\right)^{h},\left(\left(\nabla_{\partial_{i}} \partial_{k}\right)^{v}\right)\right. \\
& =c \partial_{i} g_{j k}-c g\left(\nabla_{\partial_{i}} \partial_{j}, \partial_{k}\right)-c g\left(\partial_{j}, \nabla_{\partial_{i}} \partial_{k}\right), \\
& =c \nabla_{i} g_{j k}, \\
& \left(\nabla_{\partial_{\bar{i}}}^{h} G_{a, b, c}\right)\left(\delta_{j}, \delta_{k}\right)=\partial_{\bar{i}} G_{a, b, c}\left(\delta_{j}, \delta_{k}\right)-G_{a, b, c}\left(\nabla_{\bar{\partial}_{\bar{i}}}^{h} \delta_{j}, \delta_{k}\right)-G_{a, b, c}\left(\delta_{j}, \nabla_{\overline{\bar{i}}_{\bar{i}}}^{h} \delta_{k}\right) \\
& =\partial_{\bar{i}}(a+c) g_{j k}=0, \\
& \left(\nabla_{\bar{\partial}_{\bar{i}}}^{h} G_{a, b, c}\right)\left(\delta_{j}, \partial_{\bar{k}}\right)=\partial_{\bar{i}} G_{a, b, c}\left(\delta_{j}, \partial_{\bar{k}}\right)-G_{a, b, c}\left(\nabla_{\partial_{\bar{i}}}^{h} \delta_{j}, \partial_{\bar{k}}\right)-G_{a, b, c}\left(\delta_{j}, \nabla_{\partial_{\bar{i}}}^{h} \partial_{\bar{k}}\right) \\
& =\partial_{\bar{i}} b g_{j k}=0, \\
& \left(\nabla_{\partial_{\bar{i}}}^{h} G_{a, b, c}\right)\left(\partial_{\bar{j}}, \partial_{\bar{k}}\right)=\partial_{\bar{i}} G_{a, b, c}\left(\partial_{\bar{j}}, \partial_{\bar{k}}\right)-G_{a, b, c}\left(\nabla_{\partial_{\bar{i}}}^{h} \partial_{\bar{j}}, \partial_{\bar{k}}\right)-G_{a, b, c}\left(\partial_{\bar{j}}, \nabla_{\partial_{\bar{i}}}^{h} \partial_{\bar{k}}\right) \\
& =\partial_{\bar{i}} c g_{j k}=0 \\
& =0
\end{aligned}
$$

Theorem 3.1. Let $\nabla$ be an arbitrary linear connection on a Riemannian manifold $(M, g)$ and $T M$ be its tangent bundle with the Riemannian metric $G_{a, b, c}$. The pair $\left(\nabla^{h}, G_{a, b, c}\right)$ is a Codazzi pair on TM if and only if $\nabla$ is a metric connection with respect to $g$.

Proof. Using conditions being Codazzi pair on $T M$ and (3.2) and (3.4), we immediately find

equivalently,

$$
\left(\nabla_{\delta_{i}}^{h} G_{a, b, c}\right)\left(\delta_{j}, \partial_{\bar{k}}\right)=\left(\nabla_{\delta_{j}}^{h} G_{a, b, c}\right)\left(\delta_{i}, \partial_{\bar{k}}\right)=\left(\nabla_{\partial_{\bar{k}}}^{h} G_{a, b, c}\right)\left(\delta_{j}, \delta_{i}\right)
$$

$$
b \nabla_{i} g_{j k}=b \nabla_{i} g_{j k}=0
$$

which gives the result.

Now, we consider a particular case: the tensor $G_{a, 0,0}=a g^{v}$. In this case, we have the following result.

Theorem 3.2. Let $\nabla$ be an arbitrary linear connection on a Riemannian manifold $(M, g)$ and $T M$ be its tangent bundle with the tensor $G_{a, 0,0}=a g^{v}$. The pair $\left(\nabla^{h}, G_{a, 0,0}\right)$ is a Codazzi pair on $T M$ if and only if the pair $(\nabla, g)$ is a Codazzi pair on $M$.

Proof. For the case $c=0, b=0$, and $a \neq 0$, using (3.1)-(3.6), we get

$$
\left(\nabla_{\delta_{i}}^{h} G_{a, 0,0}\right)\left(\delta_{j}, \delta_{k}\right)=a \nabla_{i} g_{j k}
$$

all other being zero. Hence, using the Codazzi equation on $T M$, we derive

$$
\left(\nabla_{\delta_{i}}^{h} G_{a, 0,0}\right)\left(\delta_{j}, \delta_{k}\right)=\left(\nabla_{\delta_{j}}^{h} G_{a, 0,0}\right)\left(\delta_{i}, \delta_{k}\right)=\left(\nabla_{\delta_{k}}^{h} G_{a, 0,0}\right)\left(\delta_{j}, \delta_{i}\right)
$$


equivalently,

$$
a \nabla_{i} g_{j k}=a \nabla_{j} g_{i k}=a \nabla_{k} g_{j i},
$$

which completes the proof.

Next, we will study conditions that $\left(\nabla^{h}, g_{C G}\right)$ is a Codazzi pair on $T M$.

Theorem 3.3. Let $\nabla$ be an arbitrary linear connection on an $n$-dimensional $(n>1)$ Riemannian manifold $(M, g)$ and $T M$ be its tangent bundle with the Cheeger-Gromoll metric $g_{C G}$. Let $(\nabla, g)$ be a Codazzi pair on $M$, then $\left(\nabla^{h}, g_{C G}\right)$ is not a Codazzi pair on $T M$.

Proof. Let $(M, g)$ be an $n$-dimensional $(n>1)$ Riemannian manifold. Assume that $\left(\nabla^{h}, g_{C G}\right)$ is a Codazzi pair on $T M$. Using the Codazzi equation on $T M$, we derive

$$
\begin{aligned}
\left(\nabla_{\delta_{i}}^{h} g_{C G}\right)\left(\delta_{j}, \partial_{\bar{k}}\right) & =\left(\nabla_{\partial_{\bar{k}}}^{h} g_{C G}\right)\left(\delta_{i}, \delta_{j}\right) \\
& =\left(\nabla_{\delta_{j}}^{h} g_{C G}\right)\left(\partial_{\bar{k}}, \delta_{i}\right)=0, \\
\left(\nabla_{\bar{\partial}_{\bar{i}}}^{h} g_{C G}\right)\left(\partial_{\bar{j}}, \delta_{k}\right) & =\left(\nabla_{\partial_{\bar{j}}}^{h} g_{C G}\right)\left(\delta_{k}, \partial_{\bar{i}}\right) \\
& =\left(\nabla_{\delta_{k}}^{h} g_{C G}\right)\left(\partial_{\bar{i}}, \partial_{\bar{j}}\right)=0 .
\end{aligned}
$$

Also, the direct computations give

$$
\begin{array}{r}
\left(\nabla_{\delta_{i}}^{h} g_{C G}\right)\left(\delta_{j}, \delta_{k}\right)=\nabla_{i} g_{j k}, \\
\left(\nabla_{\partial_{\bar{i}}}^{h} g_{C G}\right)\left(\partial_{\bar{j}}, \partial_{\bar{k}}\right)=\partial_{\bar{i}}\left(g_{C G}\right)_{\bar{j} \bar{k}}, \\
\left(\nabla_{\bar{\partial}_{\bar{j}}}^{h} g_{C G}\right)\left(\partial_{\bar{k}}, \partial_{\bar{i}}\right)=\partial_{\bar{j}}\left(g_{C G}\right)_{\bar{k} \bar{i}}, \\
\left(\nabla_{\partial_{\bar{k}}}^{h} g_{C G}\right)\left(\partial_{\bar{i}}, \partial_{\bar{j}}\right)=\partial_{\bar{k}}\left(g_{C G}\right)_{\bar{i} \bar{j}} .
\end{array}
$$

Furthermore, we have

$$
\begin{aligned}
& \partial_{\bar{i}}\left(g_{C G}\right)_{\bar{j} \bar{k}}=\frac{-2 y^{s}}{\left(1+r^{2}\right)^{2}}\left\{g_{j k} g_{s i}+y^{s} y^{t}\left(g_{j s} g_{t k} g_{s i}\right)\right\}+\frac{y^{s}}{1+r^{2}}\left(g_{s k} g_{j i}+g_{j s} g_{i k}\right), \\
& \partial_{\bar{j}}\left(g_{C G}\right)_{\bar{k} \bar{i}}=\frac{-2 y^{s}}{\left(1+r^{2}\right)^{2}}\left\{g_{k i} g_{s j}+y^{s} y^{t}\left(g_{k s} g_{t i} g_{s j}\right)\right\}+\frac{y^{s}}{1+r^{2}}\left(g_{s i} g_{k j}+g_{k s} g_{j i}\right), \\
& \partial_{\bar{k}}\left(g_{C G}\right)_{\bar{i} \bar{j}}=\frac{-2 y^{s}}{\left(1+r^{2}\right)^{2}}\left\{g_{i j} g_{s k}+y^{s} y^{t}\left(g_{i s} g_{t j} g_{s k}\right)\right\}+\frac{y^{s}}{1+r^{2}}\left(g_{s j} g_{i k}+g_{i s} g_{k j}\right) .
\end{aligned}
$$

There are two cases:

Case 1) if $\partial_{\bar{i}}\left(g_{C G}\right)_{\bar{j} \bar{k}}=\partial_{\bar{j}}\left(g_{C G}\right)_{\bar{k} \bar{i}}=\partial_{\bar{k}}\left(g_{C G}\right)_{\bar{i} \bar{j}}=0$, then from (3.7), we have

$$
\frac{-2 y^{s}}{1+r^{2}}\left\{g_{j k} g_{s i}+y^{s} y^{t}\left(g_{j s} g_{t k} g_{s i}\right)\right\}+y^{s}\left(g_{s k} g_{j i}+g_{j s} g_{i k}\right)=0 .
$$

By differentiating (3.10) with respect to $\partial_{\bar{h}}$, we get

$$
\begin{aligned}
0= & \frac{-2 \delta_{h}^{s}\left(1+r^{2}\right)+2\left(y^{n} g_{n h}\right) 2 y^{s}}{\left(1+r^{2}\right)^{2}}\left\{g_{j k} g_{s i}+y^{s} y^{t}\left(g_{j s} g_{t k} g_{s i}\right)\right\} \\
& +\frac{y^{t}\left(g_{t k} g_{j h}+g_{j t} g_{h k}\right) g_{s i}\left(-2 y^{s}\right)}{\left(1+r^{2}\right)} \\
& +\delta_{h}^{s}\left(g_{s k} g_{j i}+g_{j s} g_{i k}\right),
\end{aligned}
$$


from which

$$
\begin{aligned}
0= & \frac{-2}{\left(1+r^{2}\right)}\left\{g_{j k} g_{h i}+y^{s} y^{t}\left(g_{j h} g_{t k} g_{h i}\right)\right\} \\
& +\frac{4 y^{n} g_{n h} y^{s}}{\left(1+r^{2}\right)^{2}}\left\{g_{j k} g_{s i}+y^{s} y^{t}\left(g_{j s} g_{t k} g_{s i}\right)\right\} \\
& \frac{y^{t}\left(g_{t k} g_{j h}+g_{j t} g_{h k}\right) g_{s i}\left(-2 y^{s}\right)}{\left(1+r^{2}\right)} \\
& +\left(g_{h k} g_{j i}+g_{j h} g_{i k}\right) .
\end{aligned}
$$

The above equation holds for every $(x, y) \in T M$. If we consider the above equation for $y=0$ (zero section), we obtain

$$
-2 g_{j k} g_{h i}+\left(g_{h k} g_{j i}+g_{j h} g_{i k}\right)=0 .
$$

On multiplying above equation in $g^{i k}$, we get $2 g_{j k} \delta_{h}^{k}=g_{h k} \delta_{j}^{k}+g_{j h} \delta_{k}^{k}$. Therefore $g_{j h}=n g_{j h}$, this implies that $n=1$, and this is a contradiction.

Case 2) If $\partial_{\bar{i}}\left(g_{C G}\right)_{\bar{j} \bar{k}}=\partial_{\bar{j}}\left(g_{C G}\right)_{\bar{k} \bar{i}}=\partial_{\bar{k}}\left(g_{C G}\right)_{\bar{i} \bar{j}} \neq 0$, then by means of (3.7), (3.8) and (3.9) we have

$$
-2 g_{j k} g_{h i}+\left(g_{h k} g_{j i}+g_{j h} g_{i k}\right)=-2 g_{k i} g_{h j}+\left(g_{h i} g_{k j}+g_{k h} g_{j i}\right) .
$$

Therefore, we have $g_{j k} g_{h i}=g_{k i} g_{h j}$, and by multiplying this equation in $g^{j h}$, we get $\delta_{k}^{h} g_{h i}=$ $g_{k i} \delta_{h}^{h}$. This implies that $g_{k i}=n g_{k i}$, and so $n=1$, and this is a contradiction, i.e., $\left(\nabla^{h}, g_{C G}\right)$ is not a Codazzi pair on $T M$.

Next, we consider a torsion-free linear connection $\nabla$ on a (pseudo-)Riemannian manifold $(M, g)$. In the case, if $(\nabla, g)$ is a Codazzi pair which characterizes what is known to information geometers as statistical structures, then the manifold $M$ together with a statistical structure $(\nabla, g)$ is called a statistical manifold. The notion of a statistical manifold was originally introduced by Lauritzen [10]. Statistical manifolds are widely studied in affine differential geometry $[10,12]$ and plays a central role in information geometry.

It is known that if $\nabla$ is torsion free, its horizontal lift connection $\nabla^{h}$ has non trivial torsion. But its complate lift connection $\nabla^{c}$ is torsion free. Furthermore, if $\nabla$ is a torsion free linear connection on $M$, then $\nabla^{h}$ is torsion free if and only if $\nabla$ is flat. In this case $\nabla^{h}$ is flat and $\nabla^{h}=\nabla^{c}$. As the direct results of Theorem 3.1 and 3.2, we have the following consequences.

Corollary 3.4. Let $(M, g)$ be a (pseudo-) Riemannian manifold, $\nabla$ be a torsion free linear connection and $T M$ be its tangent bundle with the Riemannian metric $G_{a, b, c}$. Then $\left(T M, G_{a, b, c}, \nabla^{h}\right)$ is a statistical manifold if and only if $\nabla g=0$ and $\nabla$ is flat,i.e, $\left(T M, G_{a, b, c}, \nabla^{h}\right)$ reduces to the Riemannian manifold.

Corollary 3.5. Let $(M, g)$ be a (pseudo-) Riemannian manifold, $\nabla$ be a torsion free linear connection and $T M$ be its tangent bundle with the Riemannian metric $G_{a, 0,0}=a g^{v}$. The pair $\left(\nabla^{h}, G_{a, 0,0}\right)$ is a Codazzi pair on $T M$ if and only if the pair $(M, g, \nabla)$ is a flat statistical manifold. $\nabla^{c}$.

Now, we will consider the similar problems with respect to the complete lift connection

Theorem 3.6. Let $(M, g)$ be a (pseudo-) Riemannian manifold, $\nabla$ be a torsion free linear connection and $T M$ be its tangent bundle with the Riemannian metric $G_{a, b, c}$. Then $\left(T M, G_{a, b, c}, \nabla^{c}\right)$ is a statistical manifold if and only if $\nabla g=0$ and $\nabla$ is locally flat, i.e, $\nabla$ is the flat Levi-Civita connection. Furthermore $\left(T M, G_{a, b, c}, \nabla^{c}\right)$ reduces to the Riemannian manifold. 
Proof. Let $\left(T M, G_{a, b, c}, \nabla^{c}\right)$ be a statistical manifold and $\nabla$ be a torsion free linear connection. We calculate

$$
\begin{aligned}
& \left(\nabla_{\delta_{i}}^{c} G_{a, b, c}\right)\left(\delta_{j}, \partial_{\bar{k}}\right)=\delta_{i} G_{a, b, c}\left(\delta_{j}, \partial_{\bar{k}}\right)-G_{a, b, c}\left(\nabla_{\delta_{i}}^{c} \delta_{j}, \partial_{\bar{k}}\right)-G_{a, b, c}\left(\delta_{j}, \nabla_{\delta_{i}}^{c} \partial_{\bar{k}}\right) \\
& =\delta_{i} b g_{j k}-G_{a, b, c}\left(\left(\nabla_{\partial_{i}} \partial_{j}\right)^{h}+\left(R\left(y, \partial_{i}\right) \partial_{j}\right)^{v},\left(\partial_{k}\right)^{v}\right)-G_{a, b, c}\left(\left(\partial_{j}\right)^{h},\left(\left(\nabla_{\partial_{i}} \partial_{k}\right)^{v}\right)\right. \\
& =b \delta_{i} g_{j k}-b g\left(\nabla_{\partial_{i}} \partial_{j}, \partial_{k}\right)-b g\left(\partial_{j}, \nabla_{\partial_{i}} \partial_{k}\right)+a y^{s} R_{s i j k} \\
& =b \nabla_{i} g_{j k}+a y^{s} R_{s i j k}, \\
& \left(\nabla_{\partial_{\bar{k}}}^{c} G_{a, b, c}\right)\left(\delta_{i}, \delta_{j}\right)=\partial_{\bar{k}} G_{a, b, c}\left(\delta_{i}, \delta_{j}\right)-G_{a, b, c}\left(\nabla_{\partial_{\bar{k}}}^{c} \delta_{i}, \delta_{j}\right)-G_{a, b, c}\left(\delta_{i}, \nabla_{\partial_{\bar{k}}}^{c} \delta_{j}\right) \\
& =\partial_{\bar{k}}(a+c) g_{i j}=0 .
\end{aligned}
$$

From which, using the Codazzi equation on $T M$, we get

$$
\begin{aligned}
\left(\nabla_{\delta_{i}}^{c} G_{a, b, c}\right)\left(\delta_{j}, \partial_{\bar{k}}\right) & =\left(\nabla_{\partial_{\bar{k}}}^{c} G_{a, b, c}\right)\left(\delta_{i}, \delta_{j}\right), \\
b \nabla_{i} g_{j k}+a y^{s} R_{s i j k} & =0 .
\end{aligned}
$$

Operating $\partial_{\bar{h}}$ to $(3.11)$, we obtain $R_{h k i j}=0$, i.e., $(M, g, \nabla)$ is locally flat. In the case, it follows that

$$
\nabla_{i} g_{j k}=0
$$

i.e., $\nabla$ is the flat Levi-Civita connection.

Conversely, if $\nabla$ is the flat Levi-Civita connection, then $\nabla^{h}=\nabla^{c}$. Thus, the necessary condition can be easily seen via (3.1)-(3.6).

\section{Statistical structures on tangent Lie group with respect to the com- plete lift connection}

In this section, we will introduce the concept of tangent Lie group and then consider a left-invariant statistical connection $\bar{\nabla}$ on the tangent Lie group $T G$ with a left-invariant Riemannian metric $\widetilde{g}$. Firstly, we compute the Christoffel symbols of $\bar{\nabla}$ and their relations. Also we show that if $\left(T G, \widetilde{g}, \nabla^{c}\right)$ is a statistical Lie group, then $(G, g, \nabla)$ is a Riemannian Lie group, where $\nabla$ is a left-invariant torsion free linear connection. The study of geometric structures on Lie groups and corresponding Lie algebras is very important. Recently, this study extended to generalized spaces such as Hom-Lie groups and Hom-Lie algebras (see [13] for instance).

Consider a tangent bundle $T G$ over a Lie group $G$. Using the Lie group structure of $G$, we can construct a Lie group structure on $T G$ as follows:

$$
\left(x, v_{x}\right) \cdot\left(y, w_{y}\right):=\left(x y,\left(d L_{x}\right)\left(w_{y}\right)+\left(d R_{y}\right)\left(v_{x}\right)\right),
$$

for all $x, y \in G$ and $v_{x} \in T_{x} G$ and $w_{y} \in T_{y} G$. We can easily check that this multiplication is smooth and $T G$ equipped with this action has a group structure. Therefore $(T G,$.$) is a$ Lie group.

The Lie brackets of the vertical and complete lifts satisfy the following equations [3]:

$$
\left[X^{v}, Y^{v}\right]=0, \quad\left[X^{c}, Y^{c}\right]=[X, Y]^{c}, \quad\left[X^{v}, Y^{c}\right]=[X, Y]^{v} .
$$

It has been shown that the complete and vertical lifts of any left-invariant vector fields of $G$ are left-invariant vector fields on the tangent Lie group $T G$ (for more details see Proposition 1.3 of [17]). In fact, if $\left\{X_{1}, \cdots, X_{m}\right\}$ is a basis for Lie algebra $\mathfrak{g}$ of $G$, then $\left\{X_{1}^{v}, \cdots, X_{m}^{v}, X_{1}^{c}, \cdots, X_{m}^{c}\right\}$ is a basis for the Lie algebra $\tilde{\mathfrak{g}}$ of $T G$. Let us consider a left-invariant Riemannian metric $\widetilde{g}$ as follows [3]:

$$
\widetilde{g}\left(X^{c}, Y^{c}\right)=g(X, Y), \quad \widetilde{g}\left(X^{v}, Y^{v}\right)=g(X, Y), \quad \widetilde{g}\left(X^{c}, Y^{v}\right)=0,
$$

where $X, Y$ are any two left-invariant vector fields on $G$ and $g$ is a left-invariant Riemannian metric on the Lie group $G$. 
Now, we will search the statistical structures of the Riemannian manifold $(T G, \widetilde{g})$ and their relations with the statistical structure of the Riemannian manifold $(G, g)$. Let $\bar{\nabla}$ be a linear connection on the tangent Lie group $T G$. Then it can be written with respect to the basis $\left\{\partial_{i}, \partial_{\bar{i}}\right\}$ as follows:

$$
\begin{array}{ll}
\bar{\nabla}_{\partial_{i}} \partial_{j}=\bar{\Gamma}_{i j}^{k} \partial_{k}+\bar{\Gamma}_{i j}^{\bar{k}} \partial_{\bar{k}}, & \bar{\nabla}_{\partial_{i}} \partial_{\bar{j}}=\bar{\Gamma}_{i \bar{j}}^{k} \partial_{k}+\bar{\Gamma}_{i \bar{j}}^{\bar{k}} \partial_{\bar{k}}, \\
\bar{\nabla}_{\partial_{\bar{\imath}}} \partial_{j}=\bar{\Gamma}_{\bar{\imath} j}^{k} \partial_{k}+\bar{\Gamma}_{\bar{\imath} j}^{\bar{k}} \partial_{\bar{k}}, & \bar{\nabla}_{\partial_{\bar{\imath}}} \partial_{\bar{j}}=\bar{\Gamma}_{\bar{\imath} \bar{j}}^{k} \partial_{k}+\bar{\Gamma}_{\bar{\imath} \bar{j}}^{\bar{k}} \partial_{\bar{k}},
\end{array}
$$

where $\bar{\Gamma}_{A B}^{C}, A, B, C \in\{1, \cdots, n, \overline{1}, \cdots, \bar{n}\}$, are smooth functions on $T G$.

Lemma 4.1. Let $\bar{\nabla}$ be a torsion free connection on $(T G, \widetilde{g})$, then the Christoffel symbols of $\bar{\nabla}$ have the following properties:

$$
\begin{array}{rlrl}
\bar{\Gamma}_{i j}^{k} & =\bar{\Gamma}_{j i}^{k}, & & \bar{\Gamma}_{i j}^{\bar{k}}=\bar{\Gamma}_{j i}^{\bar{k}}, \\
\bar{\Gamma}_{\bar{\imath} \bar{j}}^{k}=\bar{\Gamma}_{\bar{j} \overline{\bar{l}},}^{k}, & \bar{\Gamma}_{\bar{\imath} \bar{j}}^{\bar{k}}=\bar{\Gamma}_{\bar{\jmath} \bar{l}}^{\bar{k}}, \\
\bar{\Gamma}_{\bar{\imath} j}^{k}=\bar{\Gamma}_{j \bar{\imath}}^{k}, & \bar{\Gamma}_{\bar{\imath} j}^{\bar{k}}=\bar{\Gamma}_{\bar{\jmath} \bar{\imath}}^{\bar{k}} .
\end{array}
$$

Proof. We will just prove the first relation, because others can be proven by the similar way. Via torsion freeness of $\bar{\nabla}$, we have the following

$$
\begin{aligned}
0 & =\left[\left(\partial_{i}\right)^{c},\left(\partial_{j}\right)^{c}\right]=\left[\partial_{i}, \partial_{j}\right]=\bar{\nabla}_{\partial_{i}} \partial_{j}-\bar{\nabla}_{\partial_{j}} \partial_{i} \\
& =\left(\bar{\Gamma}_{i j}^{k} \partial_{k}+\bar{\Gamma}_{i j}^{\bar{k}} \partial_{\bar{k}}\right)-\left(\bar{\Gamma}_{j i}^{k} \partial_{k}+\bar{\Gamma}_{j i}^{\bar{k}} \partial_{\bar{k}}\right) \\
& =\left(\bar{\Gamma}_{i j}^{k}-\bar{\Gamma}_{j i}^{k}\right) \partial_{k}+\left(\bar{\Gamma}_{i j}^{\bar{k}}-\bar{\Gamma}_{j i}^{\bar{k}}\right) \partial_{\bar{k}} .
\end{aligned}
$$

Definition 4.2. A connection $\nabla$ on a Lie group $G$ is said to be left-invariant if for any two left-invariant vector fields $X$ and $Y$, the field $\nabla_{X} Y$ is also left-invariant.

Proposition 4.3. Let $(G, g, \nabla)$ be a statistical Lie group with a left-invariant metric $g$ and a left-invariant connection $\nabla$. If $(T G, \widetilde{g}, \bar{\nabla})$ is a statistical Lie group with left-invariant connection $\bar{\nabla}$, then we have the following identities

$$
\begin{aligned}
& \bar{\Gamma}_{i j}^{r} g_{r k}+\bar{\Gamma}_{i k}^{\bar{r}} g_{j r}=\bar{\Gamma}_{j k}^{r} g_{r i}+\bar{\Gamma}_{j i}^{\bar{r}} g_{k r}=\bar{\Gamma}_{k i}^{r} g_{r j}+\bar{\Gamma}_{k j}^{\bar{r}} g_{i r}, \\
& \bar{\Gamma}_{i j}^{\bar{r}} g_{r k}+\bar{\Gamma}_{i \bar{k}}^{r} g_{j r}=\bar{\Gamma}_{\bar{k} i}^{r} g_{r j}+\bar{\Gamma}_{\bar{k} j}^{r} g_{i r}=\bar{\Gamma}_{j \bar{k}}^{r} g_{r i}+\bar{\Gamma}_{j i}^{\bar{r}} g_{k r}, \\
& \bar{\Gamma}_{i \bar{j}}^{\bar{r}} g_{r k}+\bar{\Gamma}_{i \bar{k}}^{\bar{r}} g_{j r}=\bar{\Gamma}_{\bar{j} \bar{k}}^{r} g_{r i}+\bar{\Gamma}_{\bar{j} i}^{\bar{r}} g_{k r}=\bar{\Gamma}_{\bar{k} i}^{\bar{r}} g_{r j}+\bar{\Gamma}_{\bar{k} \bar{j}}^{r} g_{i r}, \\
& \bar{\Gamma}_{\bar{\imath} \bar{j}}^{\bar{r}} g_{r k}+\bar{\Gamma}_{\bar{\imath} \bar{k}}^{\bar{r}} g_{j r}=\bar{\Gamma}_{\bar{j} \bar{k}}^{\bar{r}} g_{r i}+\bar{\Gamma}_{\bar{j} \bar{\imath}}^{\bar{r}} g_{k r}=\bar{\Gamma}_{\bar{j} \bar{k}}^{\bar{r}} g_{r i}+\bar{\Gamma}_{\bar{j} \bar{\imath}}^{\bar{r}} g_{k r} .
\end{aligned}
$$

Proof. Using the equation

$$
\left(\bar{\nabla}_{\partial_{i}} \widetilde{g}\right)\left(\partial_{j}, \partial_{k}\right)=-\bar{\Gamma}_{i j}^{r} g_{r k}-\bar{\Gamma}_{i k}^{\bar{r}} g_{j r}
$$

and the Codazzi equation

$$
\left(\bar{\nabla}_{\partial_{i}} \widetilde{g}\right)\left(\partial_{j}, \partial_{k}\right)=\left(\bar{\nabla}_{\partial_{j}} \widetilde{g}\right)\left(\partial_{k}, \partial_{i}\right)=\left(\bar{\nabla}_{\partial_{k}} \widetilde{g}\right)\left(\partial_{i}, \partial_{j}\right)
$$

we get (4.1). Also, the following equations

$$
\begin{aligned}
& \left(\bar{\nabla}_{\partial_{i}} \widetilde{g}\right)\left(\partial_{j}, \partial_{\bar{k}}\right)=-\bar{\Gamma}_{i j}^{\bar{r}} g_{r k}-\bar{\Gamma}_{i \bar{k}}^{r} g_{j r}, \\
& \left(\bar{\nabla}_{\partial_{j}} \widetilde{g}\right)\left(\partial_{\bar{k}}, \partial_{i}\right)=-\bar{\Gamma}_{j \bar{k}}^{r} g_{r i}-\bar{\Gamma}_{j i}^{\bar{r}} g_{k r}, \\
& \left(\bar{\nabla}_{\partial_{\bar{k}}} \widetilde{g}\right)\left(\partial_{i}, \partial_{j}\right)=-\bar{\Gamma}_{\bar{k} i}^{r} g_{r j}-\bar{\Gamma}_{\bar{k} j}^{r} g_{i r},
\end{aligned}
$$

and the Codazzi equation

$$
\left(\bar{\nabla}_{\partial_{i}} \widetilde{g}\right)\left(\partial_{j}, \partial_{\bar{k}}\right)=\left(\bar{\nabla}_{\partial_{j}} \widetilde{g}\right)\left(\partial_{\bar{k}}, \partial_{i}\right)=\left(\bar{\nabla}_{\partial_{j}} \widetilde{g}\right)\left(\partial_{\bar{k}}, \partial_{i}\right)
$$


imply (4.2). Similarly, from

and the Codazzi equation

$$
\begin{aligned}
& \left(\bar{\nabla}_{\partial_{i}} \widetilde{g}\right)\left(\partial_{\bar{j}}, \partial_{\bar{k}}\right)=-\bar{\Gamma}_{i \bar{j}}^{\bar{r}} g_{r k}-\bar{\Gamma}_{i \bar{k}}^{\bar{r}} g_{j r}, \\
& \left(\bar{\nabla}_{\partial_{\bar{j}}} \widetilde{g}\right)\left(\partial_{\bar{k}}, \partial_{i}\right)=-\bar{\Gamma}_{\bar{j} \bar{k}}^{r} g_{r i}-\bar{\Gamma}_{\bar{j} i}^{\bar{r}} g_{k r}, \\
& \left(\bar{\nabla}_{\partial_{\bar{k}}} \widetilde{g}\right)\left(\partial_{i}, \partial_{\bar{j}}\right)=-\bar{\Gamma}_{\bar{k} i}^{\bar{r}} g_{r j}-\bar{\Gamma}_{\bar{k} \bar{j}}^{r} g_{i r},
\end{aligned}
$$

$$
\left(\bar{\nabla}_{\partial_{i}} \widetilde{g}\right)\left(\partial_{\bar{j}}, \partial_{\bar{k}}\right)=\left(\bar{\nabla}_{\partial_{\bar{j}}} \widetilde{g}\right)\left(\partial_{\bar{k}}, \partial_{i}\right)=\left(\bar{\nabla}_{\partial_{\bar{k}}} \widetilde{g}\right)\left(\partial_{i}, \partial_{\bar{j}}\right)
$$

we get (4.3). Finally

$$
\left(\bar{\nabla}_{\partial_{\bar{\imath}}} \widetilde{g}\right)\left(\partial_{\bar{j}}, \partial_{\bar{k}}\right)=-\bar{\Gamma}_{\bar{\imath} j}^{\bar{r}} g_{r k}-\bar{\Gamma}_{\bar{\imath} \bar{k}}^{\bar{r}} g_{j r}
$$

and

$$
\left(\bar{\nabla}_{\partial_{\bar{\imath}}} \widetilde{g}\right)\left(\partial_{\bar{j}}, \partial_{\bar{k}}\right)=\left(\bar{\nabla}_{\partial_{\bar{j}}} \widetilde{g}\right)\left(\partial_{\bar{k}}, \partial_{\bar{\imath}}\right)=\left(\bar{\nabla}_{\partial_{\bar{k}}} \widetilde{g}\right)\left(\partial_{\bar{\imath}}, \partial_{\bar{j}}\right)
$$

give (4.4) which completes the proof.

Next, we will show that there is no linear left-invariant connection $\nabla$ on the Lie group $(G, g)$ such that $\left(\widetilde{g}, \nabla^{c}\right)$ is a Codazzi pair on $T G$.

Theorem 4.4. If $(G, g, \nabla)$ is a Lie group with a left-invariant metric $g$ and $\nabla$ be a torsion free and linear left-invariant connection, then the following statements hold:

(1) If $\left(T G, \widetilde{g}, \nabla^{c}\right)$ is a statistical Lie group, then $(G, g, \nabla)$ is a Riemannian Lie group, furthermore $\left(\widetilde{g}, \nabla^{c}\right)$ is a Codazzi pair on $T G$.

(2) If $(G, g, \nabla)$ is a non-flat Riemannian Lie group, then $\left(\widetilde{g}, \nabla^{c}\right)$ is not a Codazzi pair on $T G$.

Proof. (1) Since $\partial_{i}=\left(\partial_{i}\right)^{c}$, by using the complete lift connection $\nabla^{c}$, we compute

$$
\begin{gathered}
\left(\nabla_{\partial_{i}}^{c} \widetilde{g}\right)\left(\partial_{j}, \partial_{k}\right)=\partial_{i} \widetilde{g}\left(\partial_{j}, \partial_{k}\right)-\widetilde{g}\left(\nabla_{\partial_{i}}^{c} \partial_{j}, \partial_{k}\right)-\widetilde{g}\left(\partial_{j}, \nabla_{\partial_{i}}^{c} \partial_{k}\right) \\
=-\widetilde{g}\left(\left(\nabla_{\partial_{i}} \partial_{j}\right)^{c},\left(\partial_{k}\right)^{c}\right)-\widetilde{g}\left(\left(\partial_{j}\right)^{c},\left(\nabla_{\partial_{i}} \partial_{k}\right)^{c}\right) \\
=-g\left(\nabla_{\partial_{i}} \partial_{j}, \partial_{k}\right)-g\left(\partial_{j}, \nabla_{\partial_{i}} \partial_{k}\right) \\
=-\Gamma_{i j}^{r} g_{r k}-\Gamma_{i k}^{r} g_{j r}, \\
\left(\nabla_{\partial_{j}}^{c} \widetilde{g}\right)\left(\partial_{k}, \partial_{i}\right)=-\Gamma_{j k}^{r} g_{r i}-\Gamma_{j i}^{r} g_{k r}, \\
\left(\nabla_{\partial_{k}}^{c} \widetilde{g}\right)\left(\partial_{i}, \partial_{j}\right)=-\Gamma_{k i}^{r} g_{r j}-\Gamma_{k j}^{r} g_{i r}, \\
\left(\nabla_{\partial_{\bar{l}}}^{c} \widetilde{g}\right)\left(\partial_{\bar{j}}, \partial_{\bar{k}}\right)=\left(\nabla_{\partial_{\bar{j}}}^{c} \widetilde{g}\right)\left(\partial_{\bar{k}}, \partial_{\bar{i}}\right)=\left(\nabla_{\partial_{\bar{k}}}^{c} \widetilde{g}\right)\left(\partial_{\bar{i}}, \partial_{\bar{j}}\right)=0, \\
\left(\nabla_{\partial_{i}}^{c} \widetilde{g}\right)\left(\partial_{j}, \partial_{\bar{k}}\right)=\left(\nabla_{\partial_{j}}^{c} \widetilde{g}\right)\left(\partial_{\bar{k}}, \partial_{i}\right)=\left(\nabla_{\partial_{\bar{k}}}^{c} \widetilde{g}\right)\left(\partial_{i}, \partial_{j}\right)=0, \\
\left(\nabla_{\partial_{i}}^{c} \widetilde{g}\right)\left(\partial_{\bar{j}}, \partial_{\bar{k}}\right)=\partial_{i} \widetilde{g}\left(\partial_{\bar{j}}, \partial_{\bar{k}}\right)-\widetilde{g}\left(\nabla_{\partial_{i}}^{c} \partial_{\bar{j}}, \partial_{\bar{k}}\right)-\widetilde{g}\left(\partial_{\bar{j}}, \nabla_{\partial_{i}}^{c} \partial_{\bar{k}}\right) \\
=\widetilde{g}\left(\left(\nabla_{\partial_{i}} \partial_{j}\right)^{v}, \partial_{\bar{k}}\right)-\widetilde{g}\left(\partial_{\bar{j}},\left(\nabla_{\partial_{i}} \partial_{k}\right)^{v}\right) \\
=-g\left(\nabla_{\partial_{i}} \partial_{j}, \partial_{k}\right)-g\left(\partial_{j}, \nabla_{\partial_{i}} \partial_{k}\right) \\
=-\Gamma_{i j}^{r} g_{r k}-\Gamma_{i k}^{r} g_{j r}, \\
\left(\nabla_{\partial_{\bar{j}}}^{c} \widetilde{g}\right)\left(\partial_{\bar{k}}, \partial_{i}\right)=-\Gamma_{j i}^{r} g_{k r}, \\
\left(\nabla_{\partial_{\bar{k}}}^{c} \widetilde{g}\right)\left(\partial_{i}, \partial_{\bar{j}}\right)=-\Gamma_{k i}^{r} g_{r j} .
\end{gathered}
$$

If the Codazzi equation is verified for $T G$, it implies that $\Gamma_{i j}^{r} g_{r k}=0$, for all $i, j, k$. Therefore $(g, \nabla)$ is a Riemannian pair for $G$, furthermore the second part is clear.

(2) If $\left(\widetilde{g}, \nabla^{c}\right)$ is a Codazzi pair on $T G$, then using (4.5), and (4.6), we obtain $\Gamma_{i j}^{r} g_{r k}=0$, for all $i, j, k$. On multiplying this equation by $g^{k r}$, we get $n \Gamma_{i j}^{r}=0$, thus $\Gamma_{i j}^{r}=0$, for all $i, j$. This is a contradiction with the non-flatness of the Lie group $G$. 
Consider a left-invariant metric $\bar{g}$ on the tangent Lie group $T G$ over a Lie group $G$ with a left-invariant metric $g$ and a torsion free left invariant connection $\nabla$ defined by

$$
\bar{g}=\alpha_{i j} d x^{i} \cdot d x^{j}+2 \beta_{i j} d x^{i} \cdot d y^{j}+\gamma_{i j} d y^{i} \cdot d y^{j},
$$

where $\alpha_{i j}, \beta_{i j}, \gamma_{i j}$ are smooth functions on $T G$. Next, we want to search under what conditions $\left(\bar{g}, \nabla^{c}\right)$ is a statistical structure for the Lie group $T G$.

Theorem 4.5. Let $(G, g, \nabla)$ be a statistical Lie group, and $\nabla$ be a torsion free leftinvariant connection. Then $\left(T G, \bar{g}, \nabla^{c}\right)$ is a statistical Lie group if and only if the following identities hold:

$$
\begin{gathered}
\left(\Gamma_{i k}^{r}\right)^{c} \beta_{j r}+\left(\Gamma_{i k}^{r}\right)^{v} \alpha_{j r}=\left(\Gamma_{j k}^{r}\right)^{c} \beta_{r i}+\left(\Gamma_{j k}^{r}\right)^{v} \alpha_{r i} \\
=\left(\Gamma_{i j}^{r}\right)^{c} \beta_{r k}+\left(\Gamma_{i j}^{r}\right)^{v} \alpha_{r k}, \\
\left(\Gamma_{i k}^{r}\right)^{v} \gamma_{j r}=\left(\Gamma_{j k}^{r}\right)^{v} \gamma_{r i}=\left(\Gamma_{k i}^{r}\right)^{v} \gamma_{r j}+\left(\Gamma_{k j}^{r}\right)^{v} \gamma_{i r}=0, \\
\left(\Gamma_{i k}^{r}\right)^{v} \beta_{j r}=\left(\Gamma_{j k}^{r}\right)^{v} \beta_{i r}=\left(\Gamma_{j i}^{r}\right)^{v} \beta_{r k} .
\end{gathered}
$$

Proof. Since $(f X)^{c}=f^{c} X^{v}+f^{v} X^{c}$, and $\partial_{i}=\left(\partial_{i}\right)^{c}$, using the complete lift connection $\nabla^{c}$ we have

$$
\begin{aligned}
\left(\nabla_{\partial_{i}}^{c} \bar{g}\right)\left(\partial_{j}, \partial_{k}\right) & =\partial_{i} \bar{g}\left(\partial_{j}, \partial_{k}\right)-\bar{g}\left(\nabla_{\partial_{i}}^{c} \partial_{j}, \partial_{k}\right)-\bar{g}\left(\partial_{j}, \nabla_{\partial_{i}}^{c} \partial_{k}\right) \\
& =-\bar{g}\left(\left(\nabla_{\partial_{i}} \partial_{j}\right)^{c}, \partial_{k}\right)-\bar{g}\left(\partial_{j},\left(\nabla_{\partial_{i}} \partial_{k}\right)^{c}\right) \\
& =-\bar{g}\left(\left(\Gamma_{i j}^{r} \partial_{r}\right)^{c}, \partial_{k}\right)-\bar{g}\left(\partial_{j},\left(\Gamma_{i k}^{r} \partial_{r}\right)^{c}\right) \\
& =-\bar{g}\left(\left(\Gamma_{i j}^{r}\right)^{c} \partial_{\bar{r}}+\left(\Gamma_{i j}^{r}\right)^{v} \partial_{r}, \partial_{k}\right)-\bar{g}\left(\partial_{j},\left(\Gamma_{i k}^{r}\right)^{c} \partial_{\bar{r}}+\left(\Gamma_{i k}^{r}\right)^{v} \partial_{r}\right) \\
& =-\left(\Gamma_{i j}^{r}\right)^{c} \bar{g}\left(\partial_{\bar{r}}, \partial_{k}\right)-\left(\Gamma_{i j}^{r}\right)^{v} \bar{g}\left(\partial_{r}, \partial_{k}\right) \\
& -\left(\Gamma_{i k}^{r}\right)^{c} \bar{g}\left(\partial_{j}, \partial_{\bar{r}}\right)-\left(\Gamma_{i k}^{r}\right)^{v} \bar{g}\left(\partial_{j}, \partial_{r}\right), \\
& =-\left(\Gamma_{i j}^{r}\right)^{c} \beta_{r k}-\left(\Gamma_{i j}^{r}\right)^{v} \alpha_{r k}-\left(\Gamma_{i k}^{r}\right)^{c} \beta_{j r}-\left(\Gamma_{i k}^{r}\right)^{v} \alpha_{j r} .
\end{aligned}
$$

Here, we use $\bar{g}\left(\partial_{\bar{r}}, \partial_{k}\right)=\beta_{r k}, \bar{g}\left(\partial_{r}, \partial_{k}\right)=\alpha_{r k}$, and $\bar{g}\left(\partial_{j}, \partial_{\bar{r}}\right)=\beta_{j r}, \bar{g}\left(\partial_{j}, \partial_{r}\right)=\alpha_{j r}$. By means of the Codazzi equation of $T G$, we get (4.7). Also, we can get the following

$$
\left(\nabla_{\partial_{\bar{i}}}^{c} \bar{g}\right)\left(\partial_{\bar{j}}, \partial_{\bar{k}}\right)=\left(\nabla_{\partial_{\bar{j}}}^{c} \bar{g}\right)\left(\partial_{\bar{k}}, \partial_{\bar{i}}\right)=\left(\nabla_{\partial_{\bar{k}}}^{c} \bar{g}\right)\left(\partial_{\bar{i}}, \partial_{\bar{j}}\right)=0
$$

On the other hand, we calculate

$$
\begin{gathered}
\left(\nabla_{\partial_{\bar{\imath}}}^{c} \bar{g}\right)\left(\partial_{\bar{j}}, \partial_{k}\right)=\partial_{\bar{\imath}} \bar{g}\left(\partial_{\bar{j}}, \partial_{k}\right)-\bar{g}\left(\nabla_{\partial_{\bar{i}}}^{c} \partial_{\bar{j}}, \partial_{k}\right)-\bar{g}\left(\partial_{\bar{j}}, \nabla_{\partial_{\bar{i}}}^{c} \partial_{k}\right) \\
=-\bar{g}\left(\partial_{\bar{j}},\left(\nabla_{\partial_{i}} \partial_{k}\right)^{v}\right)=-\bar{g}\left(\partial_{\bar{j}},\left(\Gamma_{i k}^{r} \partial_{r}\right)^{v}\right) \\
=-\left(\Gamma_{i k}^{r}\right)^{v} \bar{g}\left(\partial_{\bar{j}}, \partial_{\bar{r}}\right)=-\left(\Gamma_{i k}^{r}\right)^{v} \gamma_{j r}, \\
\left(\nabla_{\partial_{\bar{j}}}^{c} \bar{g}\right)\left(\partial_{k}, \partial_{\bar{i}}\right)=-\left(\Gamma_{j k}^{r}\right)^{v} \gamma_{r i}, \\
\left(\nabla_{\partial_{k}}^{c} \bar{g}\right)\left(\partial_{\bar{i}}, \partial_{\bar{j}}\right)=-\left(\Gamma_{k i}^{r}\right)^{v} \gamma_{r j}-\left(\Gamma_{k j}^{r}\right)^{v} \gamma_{i r} .
\end{gathered}
$$

Again from the Codazzi equation of $T G$, we conclude that (4.8) holds. Finally, the following

$$
\begin{aligned}
\left(\nabla_{\partial_{i}}^{c} \bar{g}\right)\left(\partial_{j}, \partial_{\bar{k}}\right) & =\partial_{i} \bar{g}\left(\partial_{j}, \partial_{\bar{k}}\right)-\bar{g}\left(\nabla_{\partial_{i}}^{c} \partial_{j}, \partial_{\bar{k}}\right)-\bar{g}\left(\partial_{j}, \nabla_{\partial_{i}}^{c} \partial_{\bar{k}}\right) \\
& =-\bar{g}\left(\left(\nabla_{\partial_{i}} \partial_{j}\right)^{c}, \partial_{\bar{k}}\right)-\bar{g}\left(\partial_{j},\left(\nabla_{\partial_{i}} \partial_{k}\right)^{v}\right) \\
& =-\bar{g}\left(\left(\Gamma_{i j}^{r} \partial_{r}\right)^{c}, \partial_{\bar{k}}\right)-\bar{g}\left(\partial_{j},\left(\Gamma_{i k}^{r} \partial_{r}\right)^{v}\right) \\
& =-\bar{g}\left(\left(\Gamma_{i j}^{r}\right)^{c} \partial_{\bar{r}}+\left(\Gamma_{i j}^{r}\right)^{v} \partial_{r}, \partial_{\bar{k}}\right)-\bar{g}\left(\partial_{j},\left(\Gamma_{i k}^{r}\right)^{v} \partial_{\bar{r}}\right) \\
& =-\left(\Gamma_{i j}^{r}\right)^{c} \bar{g}\left(\partial_{\bar{r}}, \partial_{\bar{k}}\right)-\left(\Gamma_{i j}^{r}\right)^{v} \bar{g}\left(\partial_{r}, \partial_{\bar{k}}\right)-\left(\Gamma_{i k}^{r}\right)^{v} \bar{g}\left(\partial_{j}, \partial_{\bar{r}}\right) \\
& =-\left(\Gamma_{i j}^{r}\right)^{c} \gamma_{r k}-\left(\Gamma_{i j}^{r}\right)^{v} \beta_{r k}-\left(\Gamma_{i k}^{r}\right)^{v} \beta_{j r},
\end{aligned}
$$


and

$$
\begin{aligned}
& \left(\nabla_{\partial_{j}}^{c} \bar{g}\right)\left(\partial_{\bar{k}}, \partial_{i}\right)=-\left(\Gamma_{j k}^{r}\right)^{v} \beta_{i r}-\left(\Gamma_{j i}^{r}\right)^{v} \beta_{r k}-\left(\Gamma_{j i}^{r}\right)^{c} \gamma_{k r}, \\
& \left(\nabla_{\partial_{\bar{k}}}^{c} \bar{g}\right)\left(\partial_{i}, \partial_{j}\right)=-\left(\Gamma_{k i}^{r}\right)^{v} \beta_{j r}-\left(\Gamma_{k j}^{r}\right)^{v} \beta_{i r},
\end{aligned}
$$

verify (4.9) with help of the Codazzi equation of $T G$.

It is known that for every Lie group $G$ the space of left-invariant vector fields on $G$ is a finite dimensional Lie algebra $\mathfrak{g}$ and we know that an inner product on $\mathfrak{g}$ defines a left-invariant pseudo Riemannain metric on $G$ and vice versa. Therefore the existence of a left invariant statistical connection on a Lie group $G$ is equivalent to the existence of a left-invariant connection on the Lie algebra of $G$.

In the following, we introduce a left-invariant statistical connection on a non-abelian 2 -dimensional Lie group $G$, where its Lie algebra is denoted by $\mathfrak{g}$. It is known that $\mathfrak{g}$ is 2-dimensional and non-abelian, and $\mathfrak{g}$ is generated by $X$ and $Y$ such that $[X, Y]=X$, where $X$ and $Y$ are left-invariant vector fields on $G$. We consider left-invariant Riemannian metric $g$ on the Lie algebra of $G$ as follows

$$
g(X, X)=1, g(X, Y)=0, g(Y, Y)=1 .
$$

Since $X$ and $Y$ are the basis of $\mathfrak{g}$, there are constants $A, B, C, D, E, F, G, H$ such that

$$
\begin{aligned}
& \nabla_{X} X=A X+B Y, \\
& \nabla_{X} Y=C X+D Y, \\
& \nabla_{Y} X=E X+F Y, \\
& \nabla_{Y} Y=G X+H Y .
\end{aligned}
$$

Direct computations give us

$$
\begin{aligned}
\left(\nabla_{X} g\right)(X, Y) & =X g(X, Y)-g\left(\nabla_{X} X, Y\right)-g\left(X, \nabla_{X} Y\right) \\
& =-g(A X+B Y, Y)-g(X, C X+D Y)=-B-C,
\end{aligned}
$$

and also

$$
\begin{aligned}
\left(\nabla_{Y} g\right)(X, X) & =Y g(X, X)-g\left(\nabla_{Y} X, X\right)-g\left(X, \nabla_{Y} X\right) \\
& =-2 g\left(\nabla_{Y} X, X\right)=-2 g(E X+F Y, X)=-2 E .
\end{aligned}
$$

From the Codazzi equation, we obtain $B+C=2 E$. On the other hand we have

$$
\begin{aligned}
\left(\nabla_{Y} g\right)(Y, X) & =Y g(Y, X)-g\left(\nabla_{Y} Y, X\right)-g\left(Y, \nabla_{Y} X\right) \\
& =-g(G X+H Y, X)-g(Y, E X+F Y)=-G-F,
\end{aligned}
$$

and

$$
\begin{aligned}
\left(\nabla_{X} g\right)(Y, Y) & =X g(Y, Y)-g\left(\nabla_{X} Y, Y\right)-g\left(Y, \nabla_{X} Y\right) \\
& =-2 g\left(\nabla_{X} Y, Y\right)=-2 g(C X+D Y, Y)=-2 D .
\end{aligned}
$$

From the Codazzi equation, we obtain $2 D=G+F$. Since $\nabla$ is torsion free, we deduce that

$$
X=[X, Y]=\nabla_{X} Y-\nabla_{Y} X=(C X+D Y)-(E X+F Y)=(C-E) X+(D-F) Y,
$$

therefore, $C=1+E, D=F$. According to above equations we deduce that $F=G=D$ and $C=B+2$ and $E=B+1$. Hence, the all left-invariant statistical connections on a 2-dimensional non-abelian Lie group $G$ are the following form

$$
\begin{aligned}
& \nabla_{X} X=A X+B Y, \\
& \nabla_{X} Y=(B+2) X+D Y, \\
& \nabla_{Y} X=(B+1) X+D Y, \\
& \nabla_{Y} Y=D X+H Y .
\end{aligned}
$$


Proposition 4.6. There are infinite left-invariant statistical connections on a 2 dimensional non-abelian Lie group $(G, g)$.

Now, we will show that if $(G, g)$ is a 2-dimensional non-abelian statistical Lie group with a left-invariant connection $\nabla$, then there is not any left-invariant pseudo-Riemannian metric $\bar{g}$ on $T G$ such that $\left(\bar{g}, \nabla^{c}\right)$ is a Codazzi pair for $T G$.

In the equation (4.7), if we consider $i=1, j=2, k=1$, and $i=2, j=1, k=2$, we implies that $\alpha_{11}=0$ and $\alpha_{12}=0$. In the equation (4.9), if we consider $i=1, j=2, k=2$, and $i=2, j=k=1$, we implies that $\beta_{12}=0$ and $\beta_{11}=0$. Thus, pseudo-Riemannian metric $\bar{g}$ on $T G$ is degenerate and this is a contradiction. Hence, we state the following theorem.

Theorem 4.7. There is no left-invariant pseudo-Riemannian metric $\bar{g}$ on TG such that $\left(\bar{g}, \nabla^{c}\right)$ is a Codazzi pair for $T G$, where $(G, g)$ is a 2-dimensional non-abelian Lie group equipped with a left-invariant statistical connection $\nabla$.

In the following, we introduce a family of left-invariant statistical connections on a nonabelian 3-dimensional Lie group $G$. We know that the all of non-abelian 3-dimensional Lie algebras such as $\mathfrak{g}$ are in seven classes and one of them is the Lie algebra $\tilde{\mathfrak{g}}$ generated by the orthonormal basis $X, Y, Z$ such that $[Y, Z]=X$. Now, we introduce a left-invariant statistical connection on the Lie algebra $\widetilde{\mathfrak{g}}$. We consider a left-invariant Riemannian metric $g$ on the Lie algebra $\tilde{\mathfrak{g}}$ as follows

$$
\begin{aligned}
& g(X, X)=g(Y, Y)=g(Z, Z)=1, \\
& g(X, Y)=g(X, Z)=g(Y, Z)=0 .
\end{aligned}
$$

Since $X, Y, Z$ are the basis of $\tilde{\mathfrak{g}}$, there are real constants $a_{1}, a_{2}, \cdots, c_{9}$ such that

$$
\begin{aligned}
& \nabla_{X} X=a_{1} X+a_{2} Y+a_{3} Z, \\
& \nabla_{X} Y=a_{4} X+a_{5} Y+a_{6} Z, \\
& \nabla_{X} Z=a_{7} X+a_{8} Y+a_{9} Z, \\
& \nabla_{Y} X=b_{1} X+b_{2} Y+b_{3} Z, \\
& \nabla_{Y} Y=b_{4} X+b_{5} Y+b_{6} Z, \\
& \nabla_{Y} Z=b_{7} X+b_{8} Y+b_{9} Z, \\
& \nabla_{Z} X=c_{1} X+c_{2} Y+c_{3} Z, \\
& \nabla_{Z} Y=c_{4} X+c_{5} Y+c_{6} Z, \\
& \nabla_{Z} Z=c_{7} X+c_{8} Y+c_{9} Z .
\end{aligned}
$$

Direct computations give us

$$
\begin{aligned}
& \left(\nabla_{X} g\right)(X, Y)=X g(X, Y)-g\left(\nabla_{X} X, Y\right)-g\left(X, \nabla_{X} Y\right)=-a_{2}-a_{4}, \\
& \left(\nabla_{Y} g\right)(X, X)=Y g(X, X)-g\left(\nabla_{Y} X, X\right)-g\left(X, \nabla_{Y} X\right)=-2 b_{1}, \\
& \left(\nabla_{X} g\right)(X, Z)=X g(X, Z)-g\left(\nabla_{X} X, Z\right)-g\left(X, \nabla_{X} Z\right)=-a_{3}-a_{7}, \\
& \left(\nabla_{Z} g\right)(X, X)=Z g(X, X)-g\left(\nabla_{Z} X, X\right)-g\left(X, \nabla_{Z} X\right)=-2 c_{1}, \\
& \left(\nabla_{X} g\right)(Y, Y)=X g(Y, Y)-g\left(\nabla_{X} Y, Y\right)-g\left(Y, \nabla_{X} Y\right)=-2 a_{5}, \\
& \left(\nabla_{Y} g\right)(X, Y)=Y g(X, Y)-g\left(\nabla_{Y} X, Y\right)-g\left(X, \nabla_{Y} Y\right)=-b_{2}-b_{4}, \\
& \left(\nabla_{X} g\right)(Z, Z)=X g(Z, Z)-g\left(\nabla_{X} Z, Z\right)-g\left(Z, \nabla_{X} Z\right)=-2 a_{9}, \\
& \left(\nabla_{Z} g\right)(X, Z)=Z g(X, Z)-g\left(\nabla_{Z} X, Z\right)-g\left(X, \nabla_{Z} Z\right)=-c_{3}-c_{7} .
\end{aligned}
$$




$$
\begin{aligned}
& \left(\nabla_{Y} g\right)(Z, Z)=Y g(Z, Z)-g\left(\nabla_{Y} Z, Z\right)-g\left(Z, \nabla_{Y} Z\right)=-2 b_{9} \\
& \left(\nabla_{Z} g\right)(Y, Z)=Z g(Y, Z)-g\left(\nabla_{Z} Y, Z\right)-g\left(Y, \nabla_{Z} Z\right)=-c_{6}-c_{8} . \\
& \left(\nabla_{Z} g\right)(Y, Y)=Z g(Y, Y)-g\left(\nabla_{Z} Y, Y\right)-g\left(Y, \nabla_{Z} Y\right)=-2 c_{5}, \\
& \left(\nabla_{Y} g\right)(Z, Y)=Y g(Z, Y)-g\left(\nabla_{Y} Z, Y\right)-g\left(Z, \nabla_{Y} Y\right)=-b_{8}-b_{6} .
\end{aligned}
$$

Repeatedly using the Codazzi equation we respectively deduce that

$$
\begin{aligned}
a_{2}+a_{4} & =2 b_{1}, a_{3}+a_{7}=2 c_{1}, \\
b_{2}+b_{4} & =2 a_{5}, c_{3}+c_{7}=2 a_{9}, \\
c_{6}+c_{8} & =2 b_{9}, b_{8}+b_{6}=2 c_{5} .
\end{aligned}
$$

Also, the torsion freeness of $\nabla$ implies that

$$
X=[Y, Z]=\nabla_{Y} Z-\nabla_{Z} Y=\left(b_{7}-c_{4}\right) X+\left(b_{8}-c_{5}\right) Y+\left(b_{9}-c_{6}\right) Z .
$$

Thus, we get $b_{7}=1+c_{4}, b_{8}=c_{5}, b_{9}=c_{6}$. With help of the properties of the Lie bracket and torsion freeness of $\nabla$, we get

$$
0=[X, Z]=\nabla_{X} Z-\nabla_{Z} X=\left(a_{7}-c_{1}\right) X+\left(a_{8}-c_{2}\right) Y+\left(a_{9}-c_{3}\right) Z,
$$

from which we obtain $a_{7}=c_{1}, a_{8}=c_{2}, a_{9}=c_{3}$. By $[X, Y]=0$, we deduce that $a_{4}=$ $b_{1}, a_{5}=b_{2}, a_{6}=b_{3}$. Since $\left(\nabla_{X} g\right)(Y, Z)$ is totally symmetric, we implies that $a_{6}+a_{9}=$ $b_{3}+b_{7}=c_{2}+c_{4}$. According to the above equations we implies that the following identities hold

$$
\begin{aligned}
& a_{3}=a_{7}, a_{4}=a_{2}, a_{9}=b_{7}, a_{4}=b_{1}, a_{5}=b_{2}, a_{6}=b_{3}=c_{2}-1, b_{6}=c_{5}, \\
& b_{7}=1+c_{4}, b_{8}=c_{5}, b_{9}=c_{6}, a_{7}=c_{1}, a_{8}=c_{2}, a_{9}=c_{3}=c_{7}, c_{6}=c_{8} .
\end{aligned}
$$

Hence, we have the following theorem.

Theorem 4.8. If $(G, g)$ is a non-abelian 3-dimensional Lie group with a left invariant metric $g$ such that its Lie algebra is $\widetilde{\mathfrak{g}}$ (as above), then there are infinite left-invariant statistical connections on it. In fact, there is an infinite non-abelian 3-dimensional statistical Lie group $(G, g)$ equipped with the Lie algebra $\widetilde{\mathfrak{g}}$ (as above).

Here, we will show that if $(G, g)$ is a non-abelian 3-dimensional Lie group with a leftinvariant metric $g$ and a left-invariant connection $\nabla$ such that its Lie algebra is $\widetilde{\mathfrak{g}}$ (as above), then there is no left-invariant pseudo-Riemannian metric $\bar{g}$ on $T G$ such that $\left(\bar{g}, \nabla^{c}\right)$ be a Codazzi pair for $T G$.

In the equation (4.7), if we consider $i=2, j=3, k=2$ and $i=3, j=2, k=3$, then we obtain $\alpha_{12}=\alpha_{13}=0$. Also, in the equation (4.7), if we replace $i=1, j=2, k=3$ and $i=1, j=3, k=2$, we obtain $\alpha_{11}=0$.

On the other hand, in the equation (4.9), if we consider $i=3, j=2, k=2$ and $i=2, j=3, k=3$, then we obtain $\beta_{12}=\beta_{13}=0$. Also, in the equation (4.9), if we replace $i=2, j=1, k=3$ and $i=2, j=3, k=1$, we obtain $\beta_{11}=0$. Thus, the pseudoRiemannian metric $\bar{g}$ is degenerate and this is a contradiction. Therefore, we have the following theorem.

Theorem 4.9. There is no left-invariant pseudo-Riemannian metric $\bar{g}$ on $T G$ such that $\left(\bar{g}, \nabla^{c}\right)$ is a Codazzi pair for $T G$, where $(G, g)$ is a 3-dimensional non-abelian Lie group equipped with a left invariant metric $g$ and a left-invariant statistical connection $\nabla$ such that its Lie algebra is $\tilde{\mathfrak{g}}$ (as above).

Now, we will consider 3-dimensional non-abelian Lie group $(G, g)$ with a left-invariant metric $g$ such that its Lie algebra is $\mathfrak{g}_{1}$ and it has three non zero brackets. We know that $\mathfrak{g}_{1}$ is 3-dimensional and non-abelian and generated by the orthonormal basis $X, Y, Z$ such 
that $[X, Y]=Z,[X, Z]=-Y,[Y, Z]=X$. When we use the proof of Theorem 4.8, we implies that the following identities hold

$$
\begin{aligned}
& a_{2}=a_{4}=b_{1}, a_{3}=c_{1}, a_{5}=b_{2}=b_{4}, a_{6}=1+b_{3}, a_{7}=c_{1}, \\
& a_{8}=-1+c_{2}, a_{9}=c_{3}=c_{7}, b_{6}=b_{8}=c_{5}, b_{7}=1+c_{4}, c_{8}=c_{6},
\end{aligned}
$$

which give

Theorem 4.10. If $(G, g)$ is a non-abelian 3-dimensional Lie group with a left-invariant metric $g$ such that its Lie algebra is $\mathfrak{g}_{1}$ (as above), then there are infinite left-invariant statistical connections on it. In fact, there is an infinite non-abelian 3-dimensional statistical Lie group $(G, g)$ equipped with the Lie algebra $\mathfrak{g}_{1}$ (as above).

Let us show that if $(G, g)$ is a non-abelian 3-dimensional Lie group with a left-invariant metric $g$ and a left-invariant connection $\nabla$ such that its Lie algebra is $\mathfrak{g}_{1}$ (as above), then there is no left-invariant pseudo-Riemannian metric $\bar{g}$ on $T G$ such that $\left(\bar{g}, \nabla^{c}\right)$ be a Codazzi pair for Lie group $T G$.

In equation (4.7), if we consider $i=1, j=3, k=1$, and $i=3, j=2, k=3$, we obtain $\alpha_{21}=\alpha_{13}=0$. Moreover, in the equation (4.7), if we consider $i=3, j=1, k=2$, and $i=3, j=2, k=1$, we deduce that $\alpha_{33}=0$. Therefore, the third row of $\alpha$ is zero. As the following proof for $\alpha$, we deduce that the third row of $\beta$ is zero. Thus $\bar{g}$ is degenerate and this is a contradiction. Thus, we have the following theorem.

Theorem 4.11. There is no left-invariant pseudo-Riemannian metric $\bar{g}$ on $T G$ such that $\left(\bar{g}, \nabla^{c}\right)$ be a Codazzi pair for $T G$, where $(G, g)$ is a 3-dimensional non-abelian Lie group equipped with a left invariant metric $g$ and a left-invariant statistical connection $\nabla$, such that its Lie algebra is $\mathfrak{g}_{1}$ (as above).

Here, we will consider a 3-dimensional non-abelian Lie group $(G, g)$ with a left-invariant metric $g$ such that its Lie algebra is $\mathfrak{g}_{2}$ and it has two non zero brackets. We know that $\mathfrak{g}_{2}$ is 3-dimensional and non-abelian and generated by the orthonormal basis $X, Y, Z$ such that $[X, Z]=X,[Y, Z]=X+Y$. If we follow the proof of Theorem 4.8, we see that the following identities hold

$$
\begin{aligned}
& a_{2}=a_{4}=b_{1}, a_{3}=c_{1}-1, a_{5}=b_{2}=b_{4}, a_{6}=b_{3}, a_{7}=1+c_{1}, a_{8}=c_{2}, \\
& a_{9}=c_{3}=c_{7}, b_{6}=c_{5}-1, b_{7}=1+c_{2}, b_{8}=1+c_{5}, b_{9}=c_{6}=c_{8} .
\end{aligned}
$$

Theorem 4.12. If $(G, g)$ is a non-abelian 3-dimensional Lie group with a left-invariant metric $g$ such that its Lie algebra is $\mathfrak{g}_{2}$ (as above), then there are infinite left-invariant statistical connections on it. In fact, there is an infinite non-abelian 3-dimensional statistical Lie group $(G, g)$ equipped with the Lie algebra $\mathfrak{g}_{2}$ (as above).

Finally, we will show that if $(G, g)$ is a non-abelian 3-dimensional Lie group with a left-invariant metric $g$ and a left-invariant connection $\nabla$ such that its Lie algebra is $\mathfrak{g}_{2}$ (as above), then there is no left-invariant pseudo-Riemannian metric $\bar{g}$ on $T G$ such that $\left(\bar{g}, \nabla^{c}\right)$ is a Codazzi pair for $T G$.

In the equation (4.7), if we consider $i=1, j=3, k=1$, and $i=3, j=1, k=3$, we obtain $\alpha_{11}=\alpha_{13}=0$. In addition, in the equation (4.7), if we consider $i=3, j=2, k=3$, we obtain $\alpha_{23}=0$. Finally, in the equation (4.7), if we consider $i=2, j=1, k=3$, and $i=2, j=3, k=1$, we deduce that $\alpha_{12}=0$. Therefore the first row of $\alpha$ is zero. As following the proof for $\alpha$, we deduce that the first row of $\beta$ is zero. Hence, $\bar{g}$ is degenerate and this is a contradiction. Therefore, we have the following theorem.

Theorem 4.13. There is no left-invariant pseudo-Riemannian metric $\bar{g}$ on $T G$ such that $\left(\bar{g}, \nabla^{c}\right)$ be a Codazzi pair for $T G$, where $(G, g)$ is a 3-dimensional non-abelian Lie group equipped with a left invariant metric $g$ and a left-invariant statistical connection $\nabla$, such that its Lie algebra is $\mathfrak{g}_{2}$ (as above). 


\section{References}

[1] M.T.K. Abbassi and M. Sarih, On some hereditary properties of Riemannian g-natural metrics on tangent bundles of Riemannian manifolds, Diff. Geom. Appl. 22, 19-47, 2005.

[2] S. Amari, Information geometry of the EM and em algorithms for neural networks, Neural Networks, 8 (9), 1379-1408, 1995.

[3] F. Asgari and H.R. Salimi Moghaddam, On the Riemannian geometry of tangent Lie groups, Rend. Circ. Mat. Palermo, II. Ser. 67 (2), 185-195, 2018.

[4] V. Balan, E. Peyghan and E. Sharahi, Statistical structures on the tangent bundle of a statistical manifold with Sasaki metric, Hacet. J. Math. Stat. 49 (1), 120-135, 2020.

[5] M. Belkin, P. Niyogi and V. Sindhwani, Manifold regularization: a geometric framework for learning from labeled and unlabeled examples, J. Mach. Learn. Res. 7, 23992434, 2006.

[6] L. Bilen and A. Gezer, Some results on Riemannian g-natural metrics generated by classical lifts on the tangent bundle, Eurasian Math. J. 8 (4), 18-34, 2017.

[7] T. Fei and J. Zhang, Interaction of Codazzi couplings with (Para-)Kähler geometry, Result Math. 72 (4), 2037-2056, 2017.

[8] S. Gudmundsson and E. Kappos, On the geometry of the tangent bundles, Expo. Math. 20, 1-41, 2002.

[9] S. Ianus, Statistical manifolds and tangent bundles, Sci. Bull. Univ. Politechnica of Bucharest Ser. D, 56, 29-34, 1994.

[10] S.L. Lauritzen, Statistical manifolds, In: Differential Geometry in Statistical Inferences, IMS Lecture Notes Monogr. Ser. 10, Inst. Math. Statist. Hayward California, 96-163, 1987.

[11] H. Matsuzoe and J.I. Inoguchi, Statistical structures on tangent bundles, APPS. Appl.Sci. 5 (1), 55-57, 2003.

[12] K. Nomizu and T. Sasaki, Affine Differential Geometry: Geometry of Affine Immersions, vol. 111 of Cambridge Tracts in Mathematics. Cambridge University Press, Cambridge, 1994.

[13] L. Nourmohammadifar, E. Peyghan and S. Uddin, Geometry of almost Kenmotsu Hom-Lie algebras, Quaest. Math. DOI: 10.2989/16073606.2021.1886194.

[14] C.R. Rao, Information and accuracy attainable in the estimation of statistical parameters, Bull. Calcutta Math. Soc. 37, 81-91, 1945.

[15] A. Schwenk-Schellschmidt and U. Simon, Codazzi-equivalent affine connections, Result Math. 56, 211-229, 2009.

[16] K. Sun and S. Marchand-Maillet, An information geometry of statistical manifold learning, (Proceedings of the 31st International Conference on Machine Learning (ICML-14), 1-9, 2014.

[17] K. Yano, and S. Ishihara, Tangent and cotangent bundles, Marcel Dekker, Inc., New York 1973. 